\title{
Dynamical exploration of complex states of matter in neutron stars crusts
}

\author{
Virginia de la Mota*† \\ SUBATECH and Université de Nantes, France \\ E-mail: delamota@subatech.in2p3.fr
}

\section{Maja Novak}

Institut Ruder Boskovic, Croatia

E-mail: maja.zoric@irb.hr

\section{Philippe Eudes}

SUBATECH and Université de Nantes, France

E-mail: eudes@subatech.in2p3.fr

\begin{abstract}
We investigate the occurrence of exotic structures in the outermost layers of neutron stars within the framework of a microscopic model. The nucleonic dynamics is described through a timedependent mean field approach at low temperature. In this model starting from an initial crystalline lattice of nuclei at subnuclear densities the system evolves and self-organizes in various low-lying energy structures without assumption of final shapes. The effect on the behavior of these structures due to different lattice symmetries, densities, nuclear composites and lattice perturbations are analyzed. We investigate the sensitivity of transport properties of the nuclear medium to the occurrence of non-spherical clusters.
\end{abstract}

10th Latin American Symposium on Nuclear Physics and Applications

1-6 December, 2013

Montevideo, Uruguay

\footnotetext{
*Speaker.

${ }^{\dagger}$ We remain indebted to François Sébille for many years of fruitful collaborations.
} 


\section{Introduction}

Modern nuclear physics as well as many-body theories are valuable tools which may allow us to improve our understanding of the physics of compact objects. The interest is twofold: on one side, learning about the fundamental characteristics of stellar matter, as the equation of state (EOS) and the corresponding transport properties, is expected to shed light on the nature of the star and this fact may have significant astrophysical consequences. A considerable amount of work has been done concerning macroscopic characteristics of neutron stars (NS) as the relationship between stellar masses and radii in connection with the EOS [1]. Transport properties of NS, in particular those of their crusts, are important in understanding the evolution and structure of the star. As a matter of example, the thermal conductivity should be crucial during the cooling process of the star and the stellar viscosity could be relevant in the damping of different kind of oscillation modes and in the elastic properties of the crust-core boundary as well.

On the other side, the EOS and the transport properties of nuclear matter in NS are topics in direct connexion with present perspectives of interest in heavy ion (HI) reactions. It is then very promising if valuable informations about the fundamental properties of nuclear matter could be extracted from experiments involving very asymmetric systems in terrestrial laboratories.

NS are known to be born after the collapse of supernovae explosions. After their birth they proceed to cool down below $10^{9} \mathrm{~K}$, forming a solid crust in the outermost layers where densities attain values as high as $10^{11}-10^{13} \mathrm{~g} \mathrm{~cm}^{-3}$. Even if the crust contains only a small part of the star, it plays an important role in its evolution. HI and NS crusts share basic many-body aspects due to the fact that, at the conditions of density and temperature specific to the crusts, they are essentially made of interacting nucleons. Having more neutrons than protons, many physical properties are related with the asymmetry dependence of the nuclear interaction.

In this work we focused on the microscopic physics of NS crusts through a dynamical model named DYWAN [2]. In this region, densities are just below the saturation value and the nuclear matter is said to be "frustrated": subjected to the competition of the short-range nuclear attraction and the long-range Coulomb repulsion. As a consequence of this competition nuclei belonging to the solid layers may organize dynamically forming non-spherical composites. Then, structures such as rods and slabs as well as states in which matter is completely turned inside out, forming cylindrical and planar "holes", were predicted to exist in the ground state of nuclear matter by static approaches [3]. In these models the occurrence of those structures, the so called nuclear "pasta", was obtained from energy considerations on previously defined structures. Pasta phases should appear in a well defined order with increasing density, going from spherical to cylindrical, to planar, to complex structures, to finally become uniform at the highest densities. This typical sorting of structures was confirmed by recent works [4], which have also suggested the formation of these exotic shapes during the cooling process of hot NS and in the compression stages taking place in supernova cores collapse [5]. For these reasons it should be worthwhile to perform a dynamical description of pasta formation.

The present model describing the dynamics of nuclear matter in the crust of a NS is an extension of a dynamical approach initially developed in order to describe $\mathrm{HI}$ collisions at intermediate energies [6]. In contrast to the many previous studies employing static frameworks, this model allows to simulate the dynamical processes in inhomogeneous nuclear matter using a large number of 
nucleons without any assumptions on the structure of nuclear matter. Since these structures involve a variety of low energy configurations, a pure mean field description of the nuclear dynamics has been performed. Starting from initial crystalline lattices of nuclei with different symmetries nonspherical structures occur as the result of microscopic self-organization processes. The survival of those meta-stable equilibrium structures has been checked over thousands $\mathrm{fm} / \mathrm{c}$ [2]. In this work, the influence of the isotopic compositions and of random lattice perturbations on the formation of exotic structures are studied. The shear viscosity as well as the thermal conductivity of the dense stellar material have been estimated in several works [18]-[21]. The corresponding coefficients are here calculated and compared with a semiclassical approach [8].

This contribution is organized as follows. In Section 2 the bases of the model are presented. In Section 3 we analyze the occurrence of structures in oxygen and iron lattices, the response of which to coherent and to incoherent lattice perturbations are discussed. In Section 4 the transport coefficients are calculated. In Section 5 our concluding remarks and outlooks are given.

\section{A dynamical description of the crust}

At temperatures $T<1 \mathrm{MeV}$ and densities between $10^{6} \mathrm{~g} / \mathrm{cm}^{3}$ at the surface and $3 \times 10^{13} \mathrm{~g} / \mathrm{cm}^{3}$ at the interface with the core, the stellar matter can be modeled by a neutral mixture of nuclei, electrons and free neutrons. Nuclei are expected to form a crystalline lattice immersed in a degenerate relativistic gas of electrons, which may be described by a uniform density distribution [9]. Different microscopic descriptions of NS crusts have been developed since the 80's decade. Pioneer works [3] were in the framework of the liquid drop approach. They assumed geometrical shapes of matter and used the Wigner Seitz (WS) approximation, where individual cells are replaced by uniform spherical cells centered in the sites of the overall lattice. Since then, more sophisticated selfconsistent calculations have emerged. Reference can be made to approaches in one dimension and in the Wigner-Seitz (WS) approximation [10], or in three dimensions [11], all of them confirming the occurrence of non-spherical structures. Dynamical descriptions have also been developed more recently. Among them it can be mentioned a hydrodynamic approach [12], the semi-classical Molecular Dynamics (MD) model [13], the Quantum Molecular Dynamics (QMD) [14] and the DYWAN model.

In our approach nuclei are initially located on the sites of a three dimensional lattice with periodic boundary conditions. A static selfconsistent procedure is implemented in order to prepare nuclear composites either in their ground states or in excited states according to some mechanical or thermal constraints. Different kind of lattices can be implemented so as to describe the initial crystal state. A supercell is constructed by stacking individual cells in the 3 dimensions. In the case in which a unique nuclear specie is considered the number of nucleons in the overall supercell depends on the kind of lattice we use. It is given by the general rule:

$$
N=A N_{n} n^{d}
$$

where $A$ is the mass number of the nuclear composite, $N_{n}$ is the number of nuclei in an individual cell (the primitive cell), $n$ is the number of replicas per dimension and $d$ is the dimension of the lattice. In Fig. 1 are represented the primitive cells corresponding to three types of cubic arrange- 
ments, from left to right: simple cubic cell (SCC), face centered cell (FCC) and body centered cell (BCC).

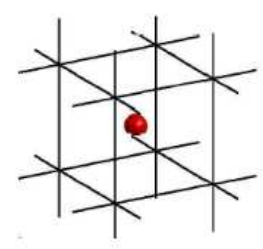

simple cubic cell

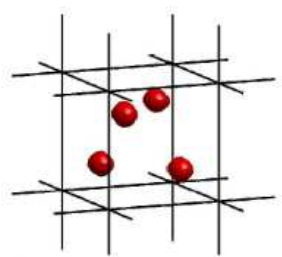

face centered cubic

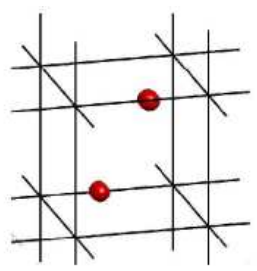

body centered cubic

Figure 1: From left to right: primitive cells corresponding to simple, face centered and body centered cubic cells.

Individual nuclei are self-consistently prepared beyond the WS approximation by solving the Hartree-Fock equation:

$$
\left[-\frac{\hbar^{2}}{2 m} \Delta+V^{H F}, \rho\right]=0
$$

To this end the one-body density matrix $\rho$ is spanned in a progressive basis of wavelets [15]. These functions, belonging to the general class of coherent states, are of the form:

$$
\alpha_{i}(x)=\mathscr{N}_{i} \mathrm{e}^{-\gamma_{1}\left(x-<x>_{i}\right)^{2}} e^{-\gamma_{2}\left(x-<x>_{i}\right)}
$$

Here $\mathscr{N}_{i}$ is a normalization coefficient and the complex numbers $\gamma_{1}$ and $\gamma_{2}$ are simple functions of first and second moments in coordinate and momentum spaces:

$$
<x>=\xi_{x} \quad<\left(x-\xi_{x}\right)^{2}>=\chi_{x} \quad<p_{x}>=\pi_{x} \quad<\left(p_{x}-\pi_{x}\right)^{2}>=\phi_{x}
$$

The momentum-position correlation $\sigma=<\frac{1}{2}\left[\left(x-\xi_{x}\right),\left(p_{x}-\pi_{x}\right)\right]_{+}>$satisfies the generalized uncertainty relation $\Delta=\chi \phi-\sigma^{2}=\frac{\hbar^{2}}{4}$. Similar expressions can be written for $y$ and $z$ components. The preceding analysis provides for each single particle level a set of 12 correlated coordinates $\{\vec{\xi}, \vec{\chi}, \vec{\pi}, \vec{\phi}\}$ representing the centroids and widths of wavelets in phase space.

For the one-body potential $V^{H F}$ in Eq. (2.1) we considered in this work a density-dependent zerorange effective interaction of the form:

$$
\begin{aligned}
V_{q}^{H F}(\rho, \xi)= & \frac{t_{0}^{\prime}}{\rho_{\infty}} \rho+\frac{t_{3}^{\prime}}{\rho_{\infty}^{v+1}} \rho^{v+1}+\frac{c}{\rho_{\infty}^{2}} \xi^{2}+\frac{4 q c}{\rho_{\infty}^{2}} \rho \xi+ \\
& \frac{\Omega}{3 \rho_{\infty}^{2}} \xi^{2}+\frac{4 q \Omega}{3 \rho_{\infty}^{2}}\left(\rho-\rho_{\infty}\right) \xi+V_{q}^{C} \\
\text { with : } \rho= & \rho_{n}+\rho_{p} \quad \xi=\rho_{n}-\rho_{p}
\end{aligned}
$$

where $\rho_{n}$ and $\rho_{p}$ stand for neutron and proton densities, $q=1 / 2$ for neutrons and $-1 / 2$ for protons, and $\rho_{\infty}=0.145 \mathrm{fm}^{-3}$. If $t_{0}, t_{3}, x_{0}$ and $x_{3}$ are the usual Skyrme effective interaction parameters [16], the ones in Eq. (2.3) satisfy the following relations: $t_{0}^{\prime}=\frac{3 \rho_{\infty}}{4} t_{0}$ and $t_{3}^{\prime}=\frac{3(v+2) \rho_{\infty}^{v+1}}{48} t_{3}$ and $x_{0}=x_{3}=-1 / 2$. The Coulomb potential $V_{q}^{C}$ has been calculated in the initial state within a Haar wavelet approximation. The current values of $c=20 \mathrm{MeV}$ and $\Omega=-100 \mathrm{MeV}$ were chosen in 
order to reproduce the typical values of baryon density energies in infinite matter [2]. The principal static characteristics of nuclei, as binding energies, radii and equilibrium densities are also correctly reproduced with this simplified force.

At the densities and excitation energies involved in the star crust the mean free path of a nucleon in the nuclear medium remains relatively long. In coherence with the building of the initial lattice, residual interactions are neglected and the systems is led to evolve according with the Time-Dependent Hartree-Fock (TDHF) equation,

$$
i \hbar \dot{\rho}=\left[-\frac{\hbar^{2}}{2 m} \Delta+V^{H F}, \rho\right]
$$

Eq. (2.4) is solved with the same mean-field as in Eq. (2.1), which is calculated using the overall density in the entire supercell. Applying a variational principle it is possible to derive [2] a set of equations of motion for wavelet centroids and widths, in each dimension:

$$
\begin{array}{cc}
\dot{\xi}=\pi / m+(\partial \mathscr{V} / \partial \pi) & \dot{\chi}=4 \gamma \chi / m-(\partial \mathscr{V} / \partial \gamma) \\
\dot{\pi}=-(\partial \mathscr{V} / \partial \xi) & \dot{\gamma}=\hbar^{2} / 8 m \chi^{2}-2 \gamma^{2} / m-(\partial \mathscr{V} / \partial \chi)
\end{array}
$$

where $\mathscr{V}=\left\langle\alpha\left|V^{H F}\right| \alpha>\right.$. The calculation of the coulomb potential $V_{q}^{C}$ is now performed using the Ewald summation techniques [17], which is adapted to the calculation of long range potentials in periodic systems.

\section{Response to coherent and to incoherent perturbations of the system}

The initial crystalline arrangement of nuclei is perturbed in order to provoke its subsequent evolution and self-organization. A coherent perturbation is a slight excitation applied identically to all nuclei. Incoherent perturbations have been introduced by slightly shifting at random the positions of nuclei from the lattice sites. Structures of the order 10 fermis are shown to appear $[2,7]$ the characteristics of which depend on the nuclear composites and on the kind of cells. In most of our primary calculations we considered mainly oxygen SCC cells for computational convenience. Nevertheless heavier nuclei as iron are expected to exist in the crust. It is well known from material science that SCC lattices are unstable against Coulomb interaction. As shown below, this is completely the case for oxygen lattices when excited with incoherent perturbations. In contrast, the system oscillates indefinitely between a few preferential shapes under the effect of coherent perturbations [2].

In Fig. 2 are represented the snapshots of oxygen isotopes with proton fraction $x=0.2$ and mean density $\langle\rho\rangle=0.058 \mathrm{fm}^{-3}$ at $t=600 \mathrm{fm} / \mathrm{c}$. On the left panel the nuclei have been initially prepared with a small quadrupole deformation long the vertical axis. On the right panel the system has the same values of $x$ and $\langle\rho\rangle$, this time the initial arrangement having been incoherently perturbed. In this case the initial symmetries are completely washed out as a result of the dynamical evolution.

Iron lattices behave in a completely different manner: they are extremely stable whatever the cellular symmetry is. In Fig. 3 we have represented the supercell density at $t=400 \mathrm{fm} / \mathrm{c}$ for an arrangement of iron isotopes with $x=0.5$ and $\langle\rho\rangle=0.058 \mathrm{fm}^{-3}$. As in Fig 2, in the left panel the system has been coherently excited at $t=0$ with a quadrupole deformation, whereas in the right one 

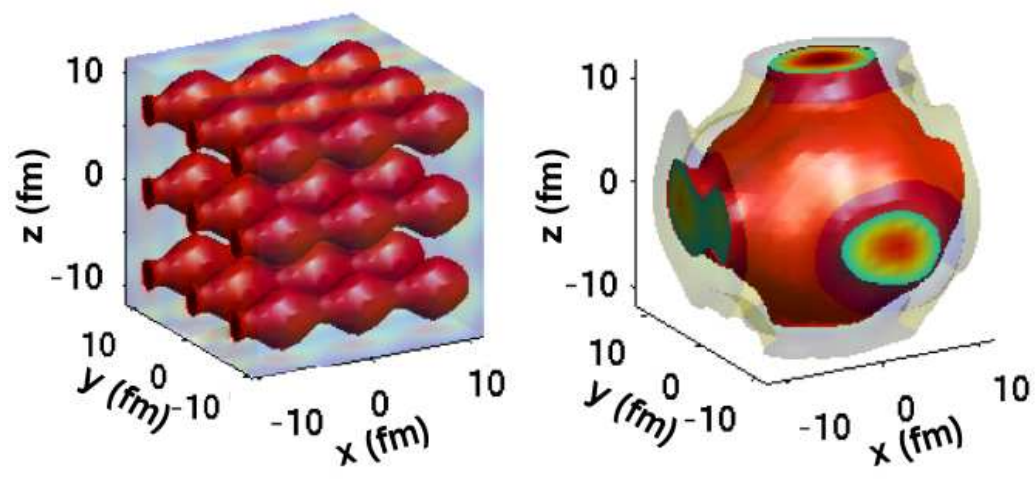

Figure 2: Oxygen SCC lattices with proton fraction 0.2 and mean density $0.058 \mathrm{fm}^{-3}$, excited with coherent deformation (left panel) and with incoherent perturbation (right panel).
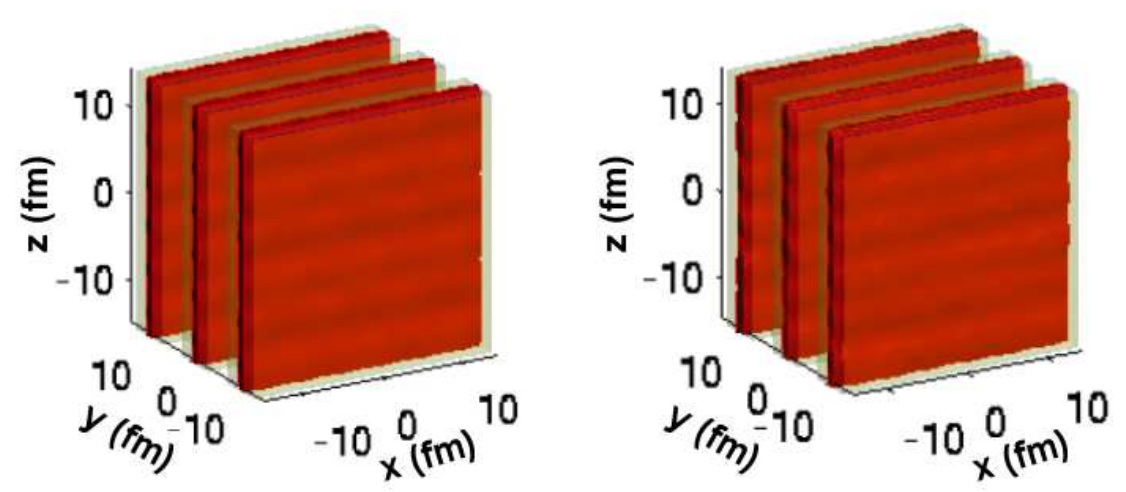

Figure 3: Iron SCC lattices with proton fraction 0.5 and mean density $0.058 \mathrm{fm}^{-3}$, excited with coherent deformation (left panel) and with incoherent perturbation (right panel).

the perturbation was incoherent. No external signs of the initial perturbation can be tracked down from the systems represented in both sides of Fig. 3.

At these high densities, proper to the inner regions of the crust, the dominant structures for the symmetric iron case are planar. More complex and stable sponge-like shapes are observed for decreasing proton fractions due to neutron dripping from clusters. The results concerning BCC lattices either for oxygen or for iron systems bear to similar conclusions, accordingly, they will not be presented here.

\section{Transport properties of the nuclear medium}

Transport properties of stellar matter are important in understanding the evolution and the structure of the star. The shear viscosity of the crust characterizes the hydrodynamic flow properties of matter in that region. It is important for a number of astrophysical problems, including the computation of the viscous damping of oscillations at the level of envelope of NS. Different kind of oscillation modes can be excited in NS which can bring important informations about their internal structure. Some oscillations, like r-modes or vortex oscillations in rapidly rotating NS, 
generate gravitational radiation. Other oscillations can be excited during X-ray bursts, which consist in nuclear explosions on the surfaces of accreting NS. High multipolarity p-mode oscillations, the corresponding restoring force being the pressure, may impact the pulse shapes of some radio pulsars. These modes may be damped to some extent depending on the shear viscosity magnitude. Inertial or r-modes of a rotating NS, the restoring force of which is the Coriolis force, may radiate gravitational waves and could limit the spin period. They can be damped according to the corresponding shear and/or bulk viscosities. On the other side the cooling time, the heat flow and the temperature distribution in the star are determined with the knowledge of the thermal conductivity of matter.

The purpose of this section is to study the transport properties of matter in the conditions of NS crusts paying a particular attention to the formation of exotic structures and their possible influence on that properties. Transport coefficients such as the shear viscosity and the thermal conductivity are estimated and compared with the results of another dynamical approach [8]. In that reference it has been argued that, as one could expect from the behavior of complex fluids, the presence of pasta phases in the nuclear medium could radically modify the corresponding transport parameters. Accordingly, we investigated the possible influence of sizes and shapes of clusters in transport coefficients, in particular looking for a possible significant modification when going from low densities regions, characterized by isolated spherical ions, to high densities where pasta phases take place.

Different authors ([18]-[21]) have calculated transport coefficients on the basis of the variational method proposed by Ziman [22] to solve the Boltzmann equation of motion for the electron distribution function. The basic hypothesis is to consider that the main carriers of momentum and energy are electrons which are scattered either by ions or by isolated protons depending on the density. Electrons are assumed to constitute a degenerate relativistic free gas while ions can be considered as a non-degenerate, non-relativistic fully ionized fluid, their corresponding mutual interaction being sufficiently weak to admit that electrons are not far from equilibrium conditions.

In coherence with Ref. [8], we implemented in our description the shear viscosity $\eta$ as derived in Ref. [20]:

$$
\eta=\frac{1}{5} n_{e} p_{F} v_{F} \tau_{\eta}
$$

where $n_{e}$ is the electron number density, $p_{F}$ and $v_{F}$ are the Fermi momentum and velocity, respectively, and $\tau_{\eta}$ the corresponding relaxation time. The thermal conductivity $\kappa$ was calculated according to Ref. [21]:

$$
\kappa=\frac{\pi^{2} n_{e} k_{B}^{2} T}{3 m^{\star}} \tau_{\kappa}
$$

where $k_{B}$ is the Boltzmann constant, $T$ is the temperature, here: $k_{B} T=1 M e V, m^{\star}=\left[m_{e}^{2}+\left(p_{F} / c\right)^{2}\right]^{1 / 2}$ is the electron relativistic effective mass, and $\tau_{\kappa}$ the characteristic relaxation time extracted from the variational method for the thermal conductivity calculation. Both relaxation times are related to the corresponding Coulomb logarithms $[19,20]$ which, in the Born approximation and in the limit of high temperatures [21], can be given in terms of the static structure function $S(k)$ of the ions. As the density increases nuclei start to melt forming complex structures, accordingly, in the same way as in Ref. [8], we assumed that the scattering processes involve nucleon coordinates and the 
Coulomb logarithm is the one describing electron-proton scattering.

In order to estimate transport coefficients within our approach we have prepared SCC and FCC lattices of iron isotopes with proton fractions $x=0.3$ and 0.5 , for mean densities in the range 0.01 $\mathrm{fm}^{-3} \leq\langle\rho\rangle \leq 0.13 \mathrm{fm}^{-3}$. As shown in the preceding section, iron lattices are stable against coherent or incoherent perturbations whatever the symmetry of the cell is. The corresponding snapshots of $x=0.3 \mathrm{FCC}$ iron supercells for different values of the average density are shown in Fig. 4. The occurrence of structures in the case $x=0.5$ as well as in SCC lattices is thoroughly resembling. At the lower density (a) $\rho=0.015 \mathrm{fm}^{-3}$ spheroidal nuclei are deformed along the vertical axis, at the intermediate value (b) $\rho=0.068 \mathrm{fm}^{-3}$ nuclei form horizontal rod-like structures and at the highest values (c) around $\rho=0.120 \mathrm{fm}^{-3}$ slab-like structures are formed.

a)

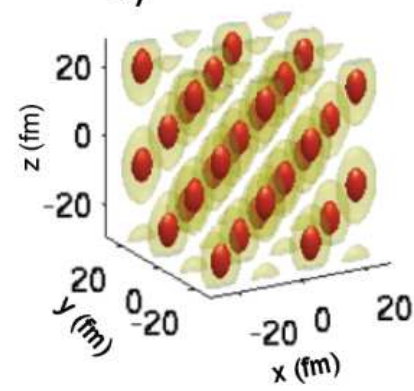

b)

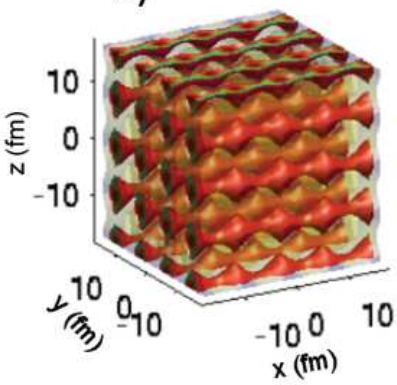

c)

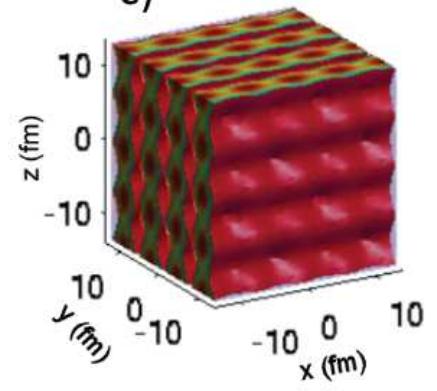

Figure 4: FCC iron lattices with proton fraction 0.3 for three values of the average density: a) $0.015, b$ ) 0.068 and c) $0.120 \mathrm{fm}^{-3}$.

The associated transport coefficients are depicted in Fig. 5. On the left panel of Fig. 5 is represented $\eta$, the shear viscosity of the iron system, for different isotopic compositions as a function of the overall mean density. In green circles are represented the values for the composite with proton fraction $x=0.5$, stars represent the results in the case $x=0.3$ and in red squares we have plotted de values extracted from Ref. [8] corresponding to a proton fraction $x=0.2$. These last calculations have been performed in the framework of the MD model. On the right side of Fig. 5 is plotted the thermal conductivity $\kappa$ with the same conventions used on the left panel.

In the case of iron SCC lattices the results do not substantially change from those in Fig. 5. Moreover, the calculated $\eta$ and $\kappa$ values are of the same order of magnitude of those of Ref. [8]. In correspondence with MD results an increase of the shear viscosity with increasing density is observed. As shown in Fig. 4 different pasta configurations follow with growing densities. Nevertheless the corresponding enhancement of both transport coefficients is far from being critical as expected [8] with the occurrence of structural transitions. Even more, the calculated $\eta$ values are only slightly higher than those of Ref. [20] which have been extracted for lower temperatures and in the absence of any non-spherical structures.

Both figures exhibit very high values of $\eta$ and $\kappa$ compared with current values of terrestrial fluids and with ordinary stars like the sun [23], which is not surprising since the corresponding temperature and density conditions are clearly not in the same range. It should be worthwhile to 

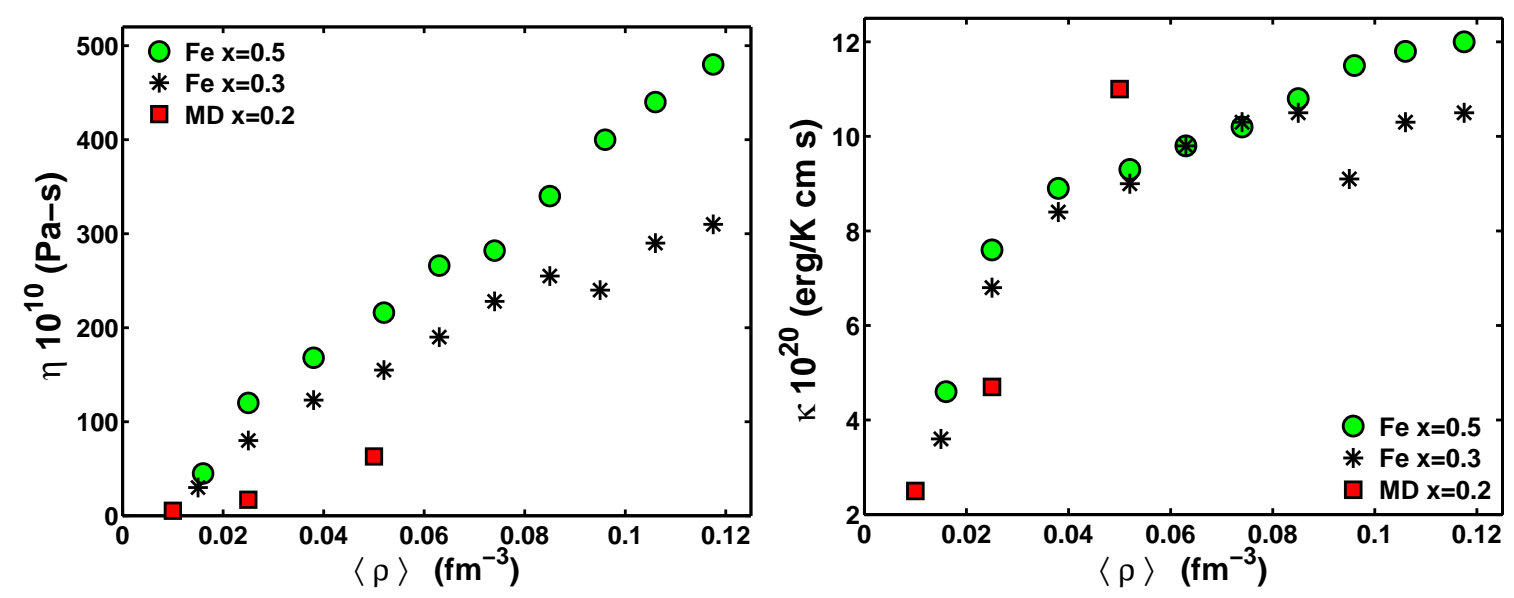

Figure 5: Transport coefficients as a function of the mean density in FCC cells with proton fraction 0.3 (stars) and 0.5 (circles). The shear viscosity is in the left panel and the thermal conductivity in the right one. In squares are the results of Ref. [8].

perform more refined calculations implementing non-local forces, which can substantially modify the dynamics, aiming at considering the nucleonic contribution to transport phenomena as well.

\section{Conclusions}

In this work we have performed a non dissipative dynamical description of matter under similar conditions of density and temperature as in neutron star crusts. In this approach a small initial amount of energy is deposited either as deformation or as random shifts in nuclei positions. The subsequent evolution, ruled by the selfconsistent mean-field, allows the system to explore a landscape of structures.

Different kind of lattices have been implemented in order to prepare the initial crystalline condition. For the most unstable arrangement corresponding to simple cubic lattices, the effects of random perturbations have been tested for two different nuclear composites. Random perturbations destroy the initial symmetries in the case of the light oxygen system whereas iron lattices are stable under the same conditions of density. At the considered densities, proper to the inner regions of the crust, an initial crystal of iron isotopes would melt favoring the occurrence of slab-like structures.

Transport properties of stellar matter are important in understanding evolution and structure of the star. Our goal was here to estimate the shear viscosity and the thermal conductivity of matter in the conditions of the crust in order to investigate the influence of sizes and shapes of clusters comparing with other calculations. Our conclusions reinforce those of Ref. [8] in the sense that no significant modification of transport coefficients takes place from low densities regions, where isolated spherical ions exist, to high densities, where non-spherical structures (pasta phases) are formed.

With the aim of making progress in the description of neutron star crusts several improvements must be conferred to the model. A more sophisticated effective force must be implemented in order to give a good description of nuclei not only at their initial states but also during their dynamical evolution. The force should at least be non-local, isospin-dependent and include asymmetry and 
spin-orbit terms. Another important aspect is the possible influence of dissipative effects on pasta formation and on the corresponding transport properties. It should be worthwhile to include residual interactions providing an extended TDHF-like description of the dynamics. New estimations of transport coefficients should be useful including nucleon contribution. This work is in progress.

\section{References}

[1] J.L. Zdunick, M. Bejger and P. Haensel, Astron. Astrophys. 491 (2008) 489.

[2] F. Sébille, S. Figerou and V. de la Mota, Nucl. Phys. A 822 (2009) 51.

[3] D.G. Ravenhall, C.J. Pethick, and J.R. Wilson, Phys. Rev. Lett. 50 (1983) 2066; M. Hashimoto, H. Seki, and M. Yamada, Prog. Theor. Phys. 71 (1984) 320 .

[4] G. Watanabe et al., Phys. Rev. C (2002) 66 012801(R); C.J. Horowitz, M.A. Pérez-Garcia and J. Pieckarewicz, Phys. Rev. C 69 (2004) 045804; M. Onsi et al., Phys. Rev. C 77 (2008) 065805; T. Maruyama et al., Phys. Rev. C (1998) 657; P. Gogëlein et al., Phys. Rev. C 77 (2008) 025802.

[5] G. Watanabe and T. Maruyama, "Nuclear pasta in supernovae and neutron stars", in Neutron Stars Crust, Ed C. Bertulani and J. Piekarewicz, Nova Sience Publishers (2012).

[6] B. Jouault, F. Sébille and V. de la Mota, Nucl. Phys. A 628 (1998)119.

[7] Sébille F, de la Mota V and Figerou S (2011) Phys. Rev. C 84055801

[8] C.J. Horowitz and D.K. Berry, Phys. Rev. C 78 (2008) 035806.

[9] Horowitz C J, Hughto J, Schneider A and Berry D K 2011 Neutron Star Crust and Molecular Dynamics Simulation arXiv:1109.5095[astro-ph.SR].

[10] P. Bonche and D. Vautherin, Nucl. Phys. A 372 (1981) 496.

[11] P. Magierski, P.-H. Heenen, Phys. Rev. C 65 (2002) 045804; W.G. Newton and J.R. Stone, Phys. Rev. 79 (2009) 055801; P. Gögelein, E. N. E. van Dalen, C. Fuchs, and H.Müther, Phys. Rev. C 77(2008) 025802; B. Shuetrumpf et al. Phys. Rev. C 87 (2013) 055805.

[12] P. Magierski, A. Bulgac, Acta Phys.Polon. B 35 (2004) 1203.

[13] C. J. Horowitz, M. A. Perez-García, D. K. Berry, J. Piekarewicz, Phys. Rev. C 72 (2005) 035801; Horowitz et al., ibid 69 (2004) 045804, ibid 70 (2004) 065086.

[14] G. Watanabe, K. Sato, K. Yasuoka and E. Ebisuzaki, Phys. Rev C 66 (2002) 012801(R).

[15] M. Unser and A. Aldroubi, "Polynomial splines and wavelets: a signal processing perspective" in Wavelets: a tutorial in Theory and applications, Ed. C.K. Chui, Academic Press, New York, p: 91(1992).

[16] E. Chabanat, P. Bonche, P. Haensel, J. Meyer, R. Schaeffer, Nucl. Phys. A 635 (1998) 231.

[17] P. Ewald, Ann Phys. 64 (1921) 253.

[18] E. Flowers and N. Itho, Astrophys. J. (1976) 218.

[19] D.G. Yakovlev and V.A. Urpin, Astron. Zh. 57 (1980) 526.

[20] A.I. Chugunov and G.G. Yakovlev, Astron. Rep. 49 (2005) 724.

[21] R. Nandkumar and C.J. Pethick, Mon. Not. R. Astron. Soc. 209 (1984) 511.

[22] J. M. Ziman, Electrons and Phonons, Clarendon, Oxford (1960).

[23] F.Q. Orral and J.B. Zirker, Astroph. J. 134 (1961) 63. 